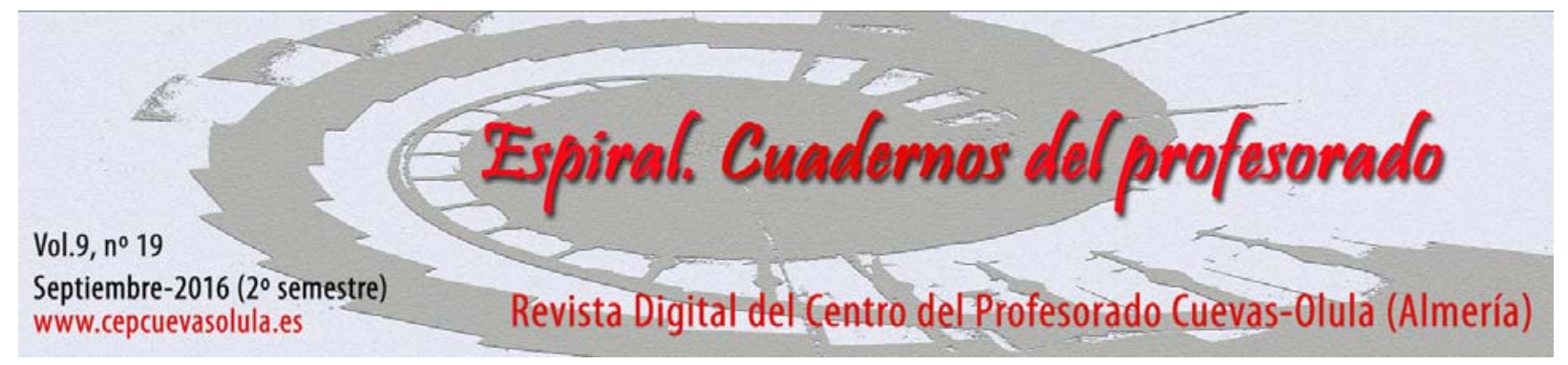

\title{
LAS COMPETENCIAS TRANSVERSALES DE LAS UNIVERSIDADES DEL CAMPUS DE EXCELENCIA AGROALIMENTARIO (CEI-A3)
}

\section{Generic skills of Cei-A3 Universities (Agrifood Campus of International Excellence)}

\section{Francisco Manzano-Agugliaro $^{(1)}$, Esther Salmerón-Manzano ${ }^{(2)}$, y Alberto Jesús Perea-Moreno $^{(3)}$}

(1) Departamento de Ingeniería, Universidad de Almería, CeiA3, España

(2) Grupo de Investigación SEJ-50. Universidad de Almería, CeiA3, España

(3) Departamento de Física Aplicada, Universidad de Córdoba, CeiA3, España

\begin{abstract}
RESUMEN: El Campus de Excelencia Internacional en Agroalimentación CeiA3 es el resultado de la integración de las Universidades de Almería, Cádiz, Huelva y Jaén, liderada por la Universidad de Córdoba. Cinco instituciones con una dilatada trayectoria científica que ponen al servicio de la sociedad su conocimiento en el sector agroalimentario con el objetivo de contribuir al desarrollo del sector. El CeiA3 cuenta con una amplia oferta de estudios agroalimentarios tanto de grado como de postgrado con unas competencias determinadas fijadas por cada universidad. El objetivo de este trabajo es realizar un análisis de las competencias transversales de las distintas universidades que componen el consorcio del CeiA3. Como resultado se observa que existen un número escaso de competencias comunes, siendo de vital importancia la incorporación de las competencias transversales comunes a los estudios universitarios de las universidades del CeiA3 ya que constituyen un elemento integrador y vertebrador del campus de excelencia internacional agroalimentario.
\end{abstract}

Palabras clave: competencias transversales, Cei-A3, universidad, excelencia.

ABSTRACT: The Agrifood Campus of International Excellence (CeiA3) is the result of the integration of the Universities of Almeria, Cadiz, Huelva and Jaen, led by the University of Cordoba. Five institutions with an extensive scientific experience that put at the service of society their knowledge in the food industry with the aim of contributing to the development of the sector. CeiA3 has a wide range of agri-food studies, at graduate levels, with skills set by each University. The aim of this paper is to carry out an analysis of the generic skills of each CeiA3 University. As a result, it was observed that there were a small number of common skills, however it is crucial to incorporate common generic skills in university studies of the CeiA3 Universities as they are an integrative and unifying element of the Agrifood Campus of International Excellence.

Key words: generic skills, Cei-A3, university, excellence.

Manzano-Agugliaro, F., Salmerón-Manzano E., y Perea-Moreno A. J. (2016). Las competencias transversales de las universidades del Campus de Excelencia Agroalimentario (Cei-A3). Espiral. Cuadernos del Profesorado, 9(19), 68-77. Disponible en: http://espiral.cepcuevasolula.es/

Fecha de recepción: 08/06/2016

Fecha de aceptación: 15/08/2016
Enviar correspondencia a: fmanzano@ual.es 


\section{Introducción}

"Las instituciones generadoras de conocimiento se han visto afectadas por los modelos de globalización, lo que ha originado un nuevo marco de competencia entre las instituciones de educación superior, que ya no afecta a entornos próximos nacionales o europeos, sino que alcanza a la totalidad del mundo universitario internacional donde la calidad de la oferta, la internacionalización de la institución y la excelencia educativa son algunos de los elementos fundamentales” (BOE, 2009).

El acuerdo del Consejo de Ministros de 30 de enero de 2009 aprobó el Plan de Acción 2009 para el apoyo y modernización de la Universidad pública española en el marco de la Estrategia Universidad 2015. El Gobierno de España lleva invertido más de 590 millones de euros desde que se puso en marcha el Programa Campus de Excelencia Internacional en 2008 (Ministerio de Educación Cultura y Deporte, 2015). Trata de promover agregaciones estratégicas entre universidades, centros de investigación, parques científicos, centros tecnológicos, entorno productivo y otros agentes, para el desarrollo de ecosistemas de educación, investigación e innovación que contribuyan al desarrollo económico y social del entorno local o regional. La idea central de estos "campus" es la creación de "ecosistemas de conocimiento" que favorezcan el empleo, la cohesión social y el desarrollo económico territorial.

En concreto, el Ministerio de Educación ha pretendido con esta iniciativa, en primer lugar, superar el hecho de la baja visibilidad global de las universidades españolas además de la atomización de las universidades mediante políticas de agregación y cooperación estratégica, lo que tiene como consecuencia una falta de capacidad para aprovechar las sinergias con su entorno y una débil política de colaboración internacional (Campos, 2010). Y en segundo lugar, es uno de los ejes estructurales de modernización de las Universidades en España que se engloba dentro de la llamada Estrategia Universidad 2015 para la modernización del Sistema universitario español, donde los proyectos de agregación en campus universitarios sean capaces de competir a nivel internacional.

El Gobierno de España ha elaborado y puesto en marcha los Campus de excelencia, para ello se ha basado en la potenciación de la Dimensión Social, la excelencia (docente, investigadora, de transferencia y de entornos) e internacionalización de las funciones de la Universidad española. En ella se plantea dar respuesta a los retos socioeconómicos de la sociedad española a través de mejoras internas, una mayor adaptación de su oferta docente, una gestión más eficiente, y una mayor contribución socioeconómica al territorio. El Plan pretende redoblar esfuerzos en formación e incentivar la generación de conocimiento para su mayor impacto en la competitividad. Dentro de dicha Estrategia, en su cuarto ámbito, el entorno, se enclava el Programa Campus de Excelencia Internacional (CEI), encaminado al desarrollo de espacios de conocimiento a través de la agregación estratégica de universidades con otros agentes del Sistema Educativo y del Sistema español de Ciencia, Tecnología y Sociedad.

En la literatura especializada, incluso hay trabajos basados en plantear que los campus de excelencia están pensados para que las universidades españolas suban en el Ranking de Shanghai (Docampo, Luque-Martínez, Torres-Salinas, y Herrera, 2012), que es actualmente el referente para conocer la posición de las universidades a nivel mundial, nos referimos al Academic Ranking of World Universities (ARWU) (Liu y Cheng, 2005). Aunque este ranking evalúa 1500 universidades de todo el mundo sólo publica las 500 mejores (http://www.shanghairanking.com).

Hay que aclarar que no existe un modelo único de campus universitario en función de los servicios e instalaciones que son necesarios para que se establezca un grado suficiente de calidad de vida académica (Vieira y Vidal, 2006). Así el propio BOE en su exposición de motivos recoge que "La interacción entre la universidad y la sociedad se produce habitualmente a través de las políticas de difusión de las actividades que se realizan en los campus con las entidades y asociaciones ciudadanas, en coordinación con las corporaciones locales y comunidades autónomas. La participación de la sociedad en la vida de los campus es hoy un reto que, entre otros elementos, 
necesita que la percepción social de la actividad universitaria vaya más allá de la formación de los nuevos graduados, asumiendo la función de responsabilidad social institucional" (BOE, 2009).

En la Tabla 1, se muestran los campus de excelencia que se crearon desde 2009 en Andalucía y la institución que los lidera. Después ha habido otras iniciativas que han conseguido financiación aunque están todavía en fase de consolidación, son los campus que todavía no están consolidados.

Tabla 1

Campus de excelencia en Andalucía

\begin{tabular}{|c|c|}
\hline Campus de excelencia & Institución que lo lidera \\
\hline Campus de Excelencia Agroalimentario (Cei-A3) & Universidad de Córdoba \\
\hline Campus Excelencia Internacional Granada (Cei Granada) & Universidad de Granada \\
\hline La Universidad de Sevilla, Campus de Excelencia Internacional & Universidad de Sevilla \\
\hline Campus de Excelencia Internacional del Mar, Ceimar & Universidad de Cádiz \\
\hline $\begin{array}{l}\text { Campus Andaluz de Excelencia Internacional en Patrimonio } \\
\text { Cultural y Natural* }\end{array}$ & Universidad de Jaén \\
\hline $\begin{array}{l}\text { Tecnologías Aplicadas al Desarrollo y a la Sostenibilidad } \\
\text { Territorial: Turismo y Hortofruticultura* }\end{array}$ & Universidad de Málaga \\
\hline
\end{tabular}

*Campus no consolidado todavía.

\section{El Campus de Excelencia Internacional en Agroalimentación CeiA3}

El Campus de Excelencia Internacional en Agroalimentación CeiA3 es el resultado de la integración de las Universidades de Almería, Cádiz, Huelva y Jaén, lideradas por la Universidad de Córdoba. Cinco instituciones con una dilatada trayectoria científica que ponen al servicio de la sociedad y el tejido productivo todo su conocimiento en el sector agroalimentario con el objetivo de contribuir al desarrollo del sector y, especialmente, a dar respuesta a los retos agroalimentarios del siglo XXI (CeiA3, 2016). Para conseguirlo, el CeiA3 pone al servicio de la sociedad los resultados del trabajo científico de más de doscientos equipos de investigación que trabajan en áreas de especial interés para el sector agroalimentario internacional, a quien quieren transferir directamente todo el conocimiento generado.

Por otra parte, y con el objetivo de seguir contribuyendo a avanzar en la generación de conocimiento, el CeiA3 cuenta con una amplia oferta de estudios agroalimentarios tanto de grado como de postgrado. En la tabla 2 se listan las titulaciones de grado incluidas dentro del CeiA3, donde se han marcado en negrita aquellas titulaciones que existen en todas las universidades del CeiA3. Se puede observar que existen titulaciones que se ofertan en todas las universidades que forman el consorcio, y no son precisamente las titulaciones relacionadas con la agricultura, sino las más demandadas por la sociedad. El objetivo de resaltar todas las titulaciones comunes es poner de manifiesto que se podrían tener competencias transversales comunes en muchas de ellas. Las actividades del CeiA3 son muy diversas incluyendo proyectos de innovación docente interuniversitarios, por ejemplo los dedicados a fomentar el bilingüismo en las carreras de Ingeniería (Manzano-Agugliaro, 2011; Manzano-Agugliaro, Sánchez y Salaberri, 2012) o actividades formativas especializadas en el campo agroalimentario como son cursos de verano.

Hay que aclarar que la pertenencia a un CEI no es excluyente de pertenecer a otros, puesto que la idea es aunar esfuerzos en una dirección concreta en la que se tiene potencial común. Por tanto no es raro que una universidad pueda tener grandes intereses y potencial para pertenecer a varios CEI a la vez. Por ejemplo la universidad de Almería pertenece al CeiA3 como se ha comentado, y también los Ceimar (liderado por Cádiz) y al CEI de Patrimonio (liderado por Jaén). 
Tabla 2

Grados ofertados por el consorcio del CeiA3

\begin{tabular}{|c|c|c|c|c|c|}
\hline GRADOS & UAL & UCA & UCO & UHU & UJA \\
\hline ADMINISTRACIÓN Y DIRECCIÓN DE EMPRESAS & $\mathbf{x}$ & $\mathbf{X}$ & $\mathbf{x}$ & $\mathbf{X}$ & $\mathbf{x}$ \\
\hline BIOLOGÍA & & & $\mathrm{X}$ & & $\mathrm{X}$ \\
\hline BIOQUÍMICA & & & $\mathrm{X}$ & & \\
\hline BIOTECNOLOGÍA & & $\mathrm{X}$ & & & \\
\hline CIENCIAS AMBIENTALES & $\mathbf{x}$ & $\mathbf{x}$ & $\mathbf{x}$ & $\mathbf{x}$ & $\mathbf{x}$ \\
\hline CIENCIAS DEL MAR & & $\mathrm{X}$ & & & \\
\hline CIENCIA Y TECNOLOGÍA DE LOS ALIMENTOS & & & $\mathrm{X}$ & & \\
\hline DERECHO & $\mathbf{x}$ & $\mathbf{X}$ & $\mathbf{x}$ & $\mathbf{x}$ & $\mathbf{x}$ \\
\hline ECONOMÍA & $\mathrm{x}$ & & & & \\
\hline ENOLOGÍA & & $\mathrm{x}$ & & & \\
\hline ESTADÍSTICA Y EMPRESA & & & & & $\mathrm{X}$ \\
\hline FINANZAS Y CONTABILIDAD & $\mathrm{x}$ & $\mathrm{X}$ & & $\mathrm{X}$ & $\mathrm{X}$ \\
\hline GEOLOGÍA & & & & $\mathrm{x}$ & \\
\hline GESTIÓN Y ADMINISTRACIÓN PÚBLICA & $\mathrm{x}$ & $\mathrm{x}$ & & & $\mathrm{X}$ \\
\hline INGENIERÍA AGRÍCOLA & $\mathrm{x}$ & & & $\mathrm{X}$ & \\
\hline $\begin{array}{l}\text { INGENIERÍA AGROALIMENTARIA Y DEL MEDIO } \\
\text { RURAL }\end{array}$ & & & $\mathrm{X}$ & & \\
\hline INGENIERÍA DE ORGANIZACIÓN INDUSTRIAL & & & & & $\mathrm{X}$ \\
\hline INGENIERÍA DE RECURSOS ENERGÉTICOS & & & & & $\mathrm{x}$ \\
\hline INGENIERÍA ELÉCTRICA & $\mathbf{x}$ & $\mathbf{x}$ & $\mathbf{x}$ & $\mathbf{X}$ & $\mathbf{x}$ \\
\hline INGENIERÍA ELECTRÓNICA INDUSTRIAL & $\mathbf{x}$ & $\mathbf{x}$ & $\mathbf{x}$ & $\mathbf{x}$ & $\mathbf{x}$ \\
\hline INGENIERÍA ENERGÉTICA & & & & $\mathrm{x}$ & \\
\hline $\begin{array}{l}\text { INGENIERÍA EN DISEÑO INDUSTRIAL Y DESARROLLO } \\
\text { DEL PRODUCTO }\end{array}$ & & $\mathrm{x}$ & & & \\
\hline INGENIERÍA EN TECNOLOGÍAS INDUSTRIALES & & $\mathrm{x}$ & & & \\
\hline $\begin{array}{l}\text { INGENIERÍA EXPLOTACIÓN DE MINAS Y RECURSOS } \\
\text { ENERGÉTICOS }\end{array}$ & & & & $\mathrm{X}$ & \\
\hline INGENIERÍA FORESTAL & & & $\mathrm{X}$ & & \\
\hline INGENIERÍA FORESTAL Y DEL MEDIO NATURAL & & & & $\mathrm{x}$ & \\
\hline INGENIERÍA GEOMÁTICA Y TOPOGRÁFICA & & & & & $\mathrm{x}$ \\
\hline INGENIERÍA INFORMÁTICA & $\mathbf{x}$ & $\mathbf{x}$ & $\mathbf{x}$ & $\mathbf{x}$ & $\mathbf{x}$ \\
\hline INGENIERÍA MECÁNICA & $\mathrm{x}$ & & & $\mathrm{x}$ & $\mathrm{x}$ \\
\hline INGENIERÍA QUÍMICA & & $\mathrm{x}$ & & & \\
\hline INGENIERÍA QUÍMICA INDUSTRIAL & $\mathrm{x}$ & & & $\mathrm{X}$ & $\mathrm{X}$ \\
\hline INGENIERÍA TELEMÁTICA & & & & & $\mathrm{X}$ \\
\hline MARKETING E INVESTIGACIÓN DE MERCADOS & $\mathrm{x}$ & $\mathrm{X}$ & & & \\
\hline MEDICINA & & $\mathrm{X}$ & $\mathrm{X}$ & & \\
\hline QUÍMICA & & $\mathrm{x}$ & $\mathrm{X}$ & $\mathrm{X}$ & $\mathrm{x}$ \\
\hline
\end{tabular}

Espiral. Cuadernos del Profesorado. ISSN 1988-7701 
PSICOLOGÍA

PUBLICIDAD Y RELACIONES PÚBLICAS

RELACIONES LABORALES Y RECURSOS HUMANOS

TRABAJO SOCIAL

TURISMO

VETERINARIA

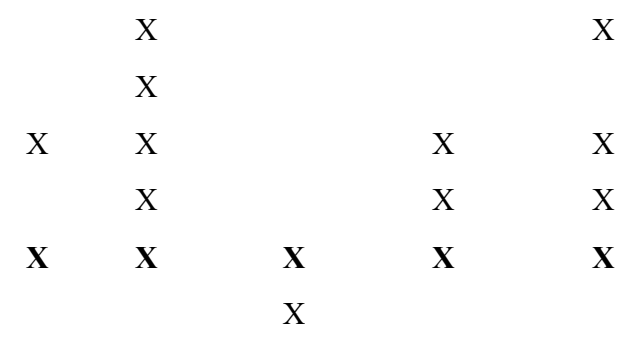

\section{Las competencias transversales o genéricas de las titulaciones}

Podemos definir las competencias como un conjunto de conocimientos, habilidades y actitudes que describen los resultados del aprendizaje de un programa educativo (Gairín et al., 2009). Dentro de estas, existen dos tipos de competencias, las específicas o técnicas básicamente relacionadas con los conocimientos técnicos de una titulación, y las competencias transversales o genéricas, que son las necesarias en un titulado con ese nivel académico, pero no necesariamente relacionadas con sus conocimientos técnicos, por ejemplo "Capacidad para aprender a trabajar de forma autónoma". Algunos autores afirman que lo importante no es la información, sino el conocimiento, ya que ciertas informaciones pueden dejar de ser válidas en un breve plazo de tiempo. Por lo tanto, se hace necesario ser capaz de buscar la información necesaria a cada momento, ser capaz de seleccionarla, procesarla, tratarla, interpretarla y apropiarse de ella para generar el conocimiento necesario que permita resolver las situaciones que se nos presenten (Cano, 2008). De ahí, la necesidad de tener por ejemplo capacidad para trabajar de forma autónoma.

Tabla 3

Competencias transversales de la Universidad de Córdoba

\begin{tabular}{ll}
\hline Código & Denominación \\
\hline CO1 & Acreditar el uso y dominio de una lengua extranjera. \\
CO2 & Conocer y perfeccionar el nivel de usuario en el ámbito de las TIC. \\
$\mathrm{CO} 3$ & Potenciar los hábitos de búsqueda activa de empleo y la capacidad de emprendimiento. \\
\hline
\end{tabular}

Las competencias transversales han sido decididas por cada universidad mediante el ejercicio de su autonomía. Estas, una vez aprobadas por el órgano competente, deben estar recogidas en todas las titulaciones de grado en al menos una asignatura. En las tablas 3, 4, 5, 6 y 7 se muestras las competencias transversales de las universidades de Córdoba, Almería, Cádiz, Jaén y Huelva respectivamente.

Tabla 4

Competencias genéricas de la Universidad de Almería

\begin{tabular}{ll}
\hline Código & Denominación \\
\hline UAL1 & Conocimientos básicos de la profesión.
\end{tabular}

UAL2 Habilidades en el uso de las TIC.

UAL3 Capacidad para resolver problemas.

UAL4 Comunicación oral y escrita en la propia lengua.

UAL5 Capacidad de crítica y autocrítica.

UAL6 Trabajo en equipo.

UAL 7 Aprendizaje de una lengua extranjera.

UAL8 Compromiso ético. 
UAL9 Capacidad para aprender a trabajar de forma autónoma.

UAL10 Competencia social y ciudadanía global.

Tabla 5

Competencias transversales de la Universidad de Cádiz

\begin{tabular}{ll}
\hline Código & Denominación \\
\hline CA1 & Trabajo en equipo. \\
CA2 & Técnicas y habilidades para la comunicación. \\
CA3 & Comportamiento en las organizaciones. \\
CA4 & Iniciativa. \\
CA5 & Aprendizaje continuo. \\
CA6 & Adaptabilidad al cambio. \\
CA7 & Capacidad de gestión. \\
CA8 & Organización y planificación. \\
CA9 & Orientación al logro y a los resultados. \\
\hline
\end{tabular}

Tabla 6

Competencias transversales de la Universidad de Jaén

Código Denominación

JA1 Ser capaz de gestionar la información y el conocimiento de su ámbito disciplinar, incluyendo saber utilizar como usuario las herramientas básicas de las tecnologías de la información y comunicación (TIC).

JA2 Localizar información concreta, explícita e implícita en textos escritos y discursos orales, organizando dicha información y construyendo conocimiento a partir de ella.

JA3 Planificar y realizar búsquedas bibliográficas o de referencias tanto en bases de datos informatizadas como en bibliotecas y o hemerotecas.

JA4 Ser capaz de proyectar los conocimientos, habilidades y destrezas adquiridos para promover una sociedad basada en los valores de la libertad, la justicia, la igualdad y el pluralismo

JA5 Capacidad para trabajar en equipo y para relacionarse con otras personas del mismo o distinto ámbito profesional.

JA6 Desarrollar habilidades de iniciación a la investigación.

Tabla 7

Competencias transversales Universidad de Huelva

\begin{tabular}{ll}
\hline Código & Denominación \\
\hline HU1 & Capacidad de análisis y síntesis. \\
HU2 & Capacidad de aprendizaje autónomo. \\
HU3 & Capacidad de comunicación oral y escrita. \\
HU4 & Conocimiento de una lengua extranjera (preferentemente inglés). \\
HU5 & Conocimientos básicos de informática (textos, hojas de cálculo, diseño gráfico, etc.).
\end{tabular}


HU6 Capacidad de resolución de problemas

HU7 Capacidad de organización y planificación.

HU8 Capacidad de gestión de información.

HU9 Capacidad de aplicar conocimientos a la práctica.

HU10 Capacidad de adaptarse a nuevas situaciones

HU11 Capacidad de toma de decisiones.

HU12 Capacidad de trabajo en grupos.

HU13 Capacidad de trabajo en equipos de carácter interdisciplinar.

HU14 Capacidad de razonamiento crítico y autocrítico.

HU15 Compromiso ético.

HU16 Motivación por la calidad.

HU17 Iniciativa y espíritu emprendedor.

\section{Análisis de las competencias transversales de las universidades del CeiA3.}

En el apartado anterior se han expuesto las competencias transversales de las distintas universidades que componen el consorcio del CeiA3, donde se puede observar la gran disparidad de número y contenido de dichas competencias. La figura 1, muestra una comparativa del número de competencias transversales de las universidades que componen el CeiA3.

Dado la pertenencia de las universidades al Campus de Excelencia internacional, cabría pensar que todas ellas incorporan en sus competencias transversales la competencia de segunda lengua, por ejemplo la CO1 "Acreditar el uso y dominio de una lengua extranjera", en sus diversas modalidades como la UAL7 "Aprendizaje de una lengua extranjera" o la HU4 "Conocimiento de una lengua extranjera (preferentemente inglés)", sin embargo, ni Jaén, ni Cádiz tienen nada referente a la competencia de segunda lengua. Esta competencia es una de las competencias más valoradas desde hace tiempo $\mathrm{y}$, en general, considerada

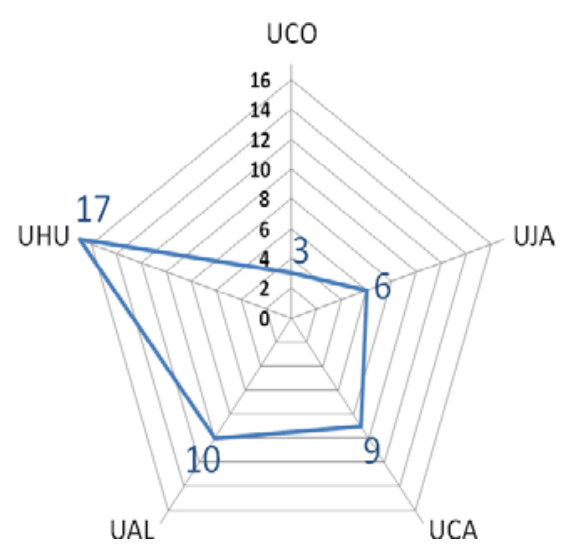
imprescindible para la inserción laboral y

Figura 1. Comparación de las competencias transversales de las universidades del CeiA3

la movilidad europea (Muñoz, 2003). En general, preocupa especialmente el aprendizaje del inglés, por eso, alguna universidad recoge especialmente este idioma en las competencias transversales como es el caso de Huelva.

Dado que las competencias pueden adoptar distintas formas o expresiones, en la tabla 8, se ha realizado una síntesis de las competencias agrupadas según la clasificación propuesta por Mir (2008), aunque existen otras como las propuestas por Cano (2005). Se observa que la competencia más frecuente es la de "Trabajo en equipo", esto es lógico teniendo en cuenta que el cambio de estructura y proceso de las organizaciones de la sociedad actual ha generado un gran impacto en la nueva manera de trabajar, donde la competencia de trabajo en equipo se impone a la individualización laboral. Esto se debe a que las tareas han aumentado en dificultad, haciendo que su resolución individual sea muy 
costosa (Torrelles et al., 2011). Por el contrario, el aprendizaje autónomo (trabajo autónomo como competencia) necesita de un gran entrenamiento (Coll, 2014), que requiere el establecimiento de metas de aprendizaje, la planificación de cursos de acción, la selección de estrategias y recursos adecuados o la persistencia en la resolución de la tarea, su revisión y reorientación para lograr alcanzar los objetivos prefijados (Mauri y Rochera, 1997).

En lo referente las competencias relacionadas con las tecnologías, las competencias informacionales y digitales, parece existir también un consenso en que el manejo de las TIC es una competencia importante, reflejándose en 4 de las 5 universidades, la definición de esta competencia puede entenderse que "implica el uso racional y crítico de la tecnología para trabajar y vivir en la

sociedad de la información e incluye las siguientes habilidades básicas: el uso del ordenador para buscar, recuperar, analizar, producir, presentar y difundir la información, así como para comunicarse y participar en redes colaborativas" (Guitert, Guerrero, Ornellas, Romeu y Romero, 2008). Por el contrario, la de búsqueda de información se refleja sólo en dos de las 5 universidades, por la propia definición que se ha indicado anteriormente puede que estén englobadas dentro de las TIC, pues es difícil hoy en día entender la búsqueda de información sin el uso de las TIC, de hecho muchos autores así lo reflejan (Valero, Aramburu, Baños, Sentí y Pérez 2007).

En el bloque de las competencias instrumentales. Se observa que estas son las menos seleccionadas por las universidades como competencias transversales, donde la más frecuente, sólo lo está en dos universidades como máximo, nos referimos a las relacionadas con "la capacidad de análisis y síntesis" o las de organizar y planificar". Esto contradice otros modelos de enseñanza como el de américa latina donde esta competencia (Análisis y síntesis) es la más valorada de todas (Bozu y Herrera, 2009), pues es considerada una competencia relacionada con el aprendizaje.

No podemos dejar de mencionar las competencias relativas a los valores, tal es el caso de la competencia del "Compromiso ético" presente sólo en 3 de las 5 universidades. Esta competencia no es una prioridad para casi todas las universidades. Otros trabajos también destacan este aspecto y ofrecen reflexiones hacia la necesidad de concebir la educación en valores como un eje transversal en la formación universitaria, lo que implica lograr un aprendizaje profesional ético y de compromiso social (González y González, 2008).

Tras el análisis realizado, se han observado grandes diferencias en las competencias transversales entre las universidades del CeiA3. Entendemos que se debería abrir un debate para para mejorar esta situación incorporando las competencias transversales de forma progresiva y coordinada. Y a ser posible dentro de las reuniones sectoriales del Cei. Por tanto el diseño de las competencias transversales debe hacerse con visión de conjunto y teniendo en cuanta la gran cantidad de titulaciones comunes entre las universidades que componen el CeiA3. Igualmente, y dado que las competencias transversales deben ser evaluadas dentro de las distintas asignaturas en los planes de estudio, habría que evitar el posible riesgo de sobrecargar de competencias, como es el caso de la universidad de Huelva que tiene 17. En el otro extremo tenemos el caso de la universidad de Córdoba con sólo 3. Por tanto, a tenor de nuestro estudio, nuestra propuesta sería un modelo de no más de 10 competencias transversales.

Tras el análisis realizado, se han observado grandes diferencias en las competencias transversales entre las universidades del CeiA3. Entendemos que se debería abrir un debate para para mejorar esta situación incorporando las competencias transversales de forma progresiva y coordinada. $\mathrm{Y}$ a ser posible dentro de las reuniones sectoriales del Cei. Por tanto el diseño de las competencias transversales debe hacerse con visión de conjunto y teniendo en cuanta la gran cantidad de titulaciones comunes entre las universidades que componen el CeiA3. Igualmente, y dado que las competencias transversales deben ser evaluadas dentro de las distintas asignaturas en los planes de estudio, habría que evitar el posible riesgo de sobrecargar de competencias, como es el caso de la universidad de Huelva que tiene 17. En el otro extremo tenemos el caso de la universidad de Córdoba con sólo 3. Por tanto, a tenor de nuestro estudio, nuestra propuesta sería un modelo de no más de 10 competencias transversales. 
Tabla 8

Agrupación de competencias transversales de las universidades del CeiA3

\begin{tabular}{|c|c|c|c|c|c|c|c|c|c|c|c|}
\hline \multirow[b]{2}{*}{ 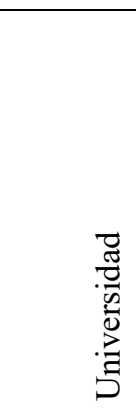 } & \multicolumn{2}{|c|}{ Instrumentales } & \multicolumn{2}{|c|}{ Comunicación } & \multicolumn{3}{|c|}{$\begin{array}{c}\text { Informacionales y } \\
\text { Digitales }\end{array}$} & \multicolumn{3}{|c|}{ Trabajo } & \multirow[b]{2}{*}{ 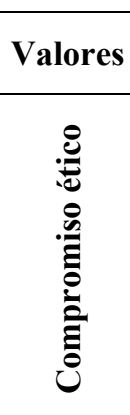 } \\
\hline & 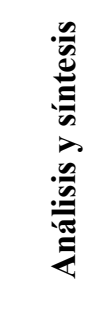 & 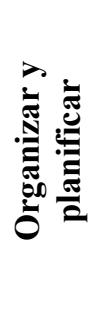 & 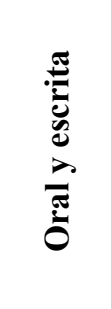 & 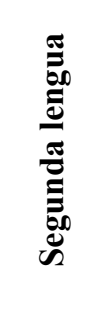 & 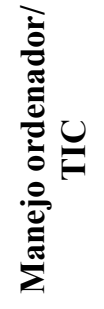 & 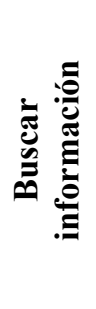 & 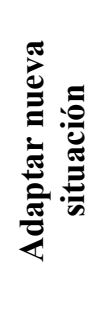 & 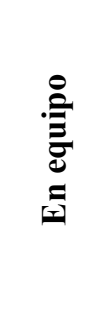 & 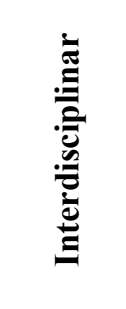 & 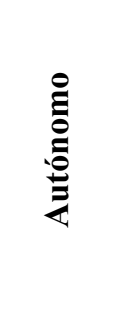 & \\
\hline Córdoba & & & & $\mathrm{CO} 1$ & $\mathrm{CO} 2$ & & & & & & \\
\hline Jaén & & & & & JA1 & $\begin{array}{l}\text { JA2- } \\
\text { JA3 }\end{array}$ & & JA5 & & & JA4 \\
\hline Cádiz & & $\begin{array}{l}\text { CA7- } \\
\text { CA8 }\end{array}$ & CA2 & & & & & CA1 & CA3 & CA5 & \\
\hline Almería & UAL5 & & UAL4 & UAL7 & UAL2 & & & UAL6 & & UAL9 & $\begin{array}{l}\text { UAL8- } \\
\text { UAL10 }\end{array}$ \\
\hline Huelva & $\begin{array}{l}\text { HU1- } \\
\text { HU14 }\end{array}$ & & HU3 & HU4 & HU5 & HU8 & HU10 & HU12 & HU13 & HU2 & HU15 \\
\hline
\end{tabular}

\section{Conclusiones}

El reto para los Campus de Excelencia en general y para el CeiA3 en particular es avanzar con paso firme hacia la situación estratégica óptima de crecimiento. Por tanto, sería recomendable orientar sus esfuerzos en aquellas líneas de actuación con mayor incidencia en el proceso competencial y, aprovechar los posibles futuros diseños de los Planes de Estudios para mejorar y enfatizar el desarrollo de las competencias transversales más valoradas, de manera coordinada entre las distintas universidades que componen el CeiA3. De este modo, la incorporación de las competencias transversales comunes a los estudios universitarios resultará de vital importancia para las universidades del CeiA3, presentándose realmente como un elemento integrador y vertebrador del campus de excelencia internacional agroalimentario en una sociedad cambiante que reformula sus demandas constantemente y que a su vez aspira a profesionalizar la formación universitaria acercando la universidad a la sociedad y al mundo laboral.

\section{Referencias}

BOE. (2009). BOLETÍN OFICIAL DEL ESTADO Jueves 23 de julio de 2009 [en línea]. Disponible en: http://www.boe.es/boe/dias/2009/07/23/pdfs/BOE-A-2009-12275.pdf [Consulta: 2016, 15 de mayo].

Bozu, Z., \& Herrera, P. J. C. (2009). El profesorado universitario en la sociedad del conocimiento: competencias profesionales docente. Revista de Formación e Innovación Educativa Universitaria (REFIEDU), 2(2), 221-231.

Campos, P. (2010). España-Campus de excelencia internacional. Spain-Campus of International Excellence. Madrid: Ministerio de Educación.

Cano García, M. E. (2008). La evaluación por competencias en la educación superior. Revista de currículum y formación del profesorado, 12(3), 1-16. [en línea]. Disponible en: http://www.ugr.es/local/recfpro/rev123COL1.pdf [Consulta: 2016, 15 de mayo].

Cano, E. (2005). El portafolios del profesorado universitario. Un instrumento para la evaluación y para el desarrollo profesional. Barcelona: Octaedro.

CeiA3 (2016). [en línea]. Disponible en: http://www.ceia3.es/ [Consulta: 2016, 15 de mayo].

Coll, C. (2014). La evaluación continuada como instrumento para eL ajuste de La ayuda pedagógica y La enseñanza de competencias de autorreguLación. Cuaderno de Pedagogía Universitaria, 8(15), 14-20. 
Docampo, D., Luque-Martínez, T., Torres-Salinas, D., \& Herrera, F. (2012). Efecto de la agregación de universidades españolas en el Ranking de Shanghai (ARWU): caso de las comunidades autónomas y los campus de excelencia. El profesional de la información, 21(4), 428-432.

Gairín, J., Armengol, C., Gisbert, M., García, M. J., Rodríguez, D., \& Cela, J. M. (2009). Guía para la evaluación de competencias en el área de Ciencias Sociales. Barcelona: Agència per a la Qualitat del Sistema Universitari de Catalunya.

González Maura, V. \& González Tirados, R. M. (2008). Competencias genéricas y formación profesional: un análisis desde la docencia universitaria. Revista Iberoamericana de educación, (47), 185-210.

Guitert, M., Guerrero, A.E., Ornellas, A., Romeu, T. y Romero, M. (2008). Implementación de la competencia transversal «Uso y aplicación de las TIC en el ámbito académico y profesional» en el contexto universitario de la UOC. Revista Latinoamericana de Tecnología Educativa, 7(2), 81-89 [en línea]. Disponible en: http://campusvirtual.unex.es/cala/editio/ [Consulta: 2016, 15 de mayo].

Liu, N. C., \& Cheng, Y. (2005). The academic ranking of world universities.Higher education in Europe, 30(2), 127-136.

Manzano-Agugliaro, F. (2011). CEIA3 Innovation Teaching Project: Bilingualism In Engineering. ICERI2011 Proceedings, 1839-1845.

Manzano-Agugliaro, F., Sánchez Pérez, M. S., \& Salaberri Ramiro, S. (2012). Bilingualism In Engineering Curricula At CeiA3 Universities. INTED2012 Proceedings, 536-542.

Mauri, T., Rochera, M.J. (1997). Aprender a regular el propio aprendizaje. Aula de Innovación Educativa, 67, 48-52.

Ministerio de Educación Cultura y Deporte. (2015). En línea, consultado Mayo 2015. [en línea]. Disponible en: http://www.mecd.gob.es/mecd/servicios-al-ciudadano-mecd/catalogo/general/educacion/0-cei/ficha/cei2009\#dg1 [Consulta: 2016, 15 de mayo].

Mir Acebrón, A. (2008). Las competencias transversales en la Universidad Pompeu Fabra. La visión de los docentes y estudiantes de segundo ciclo. Revista de Docencia Universitaria. Núm monográfico I, 1-16. [en línea]. Disponible en: http://www.redu.um.es/Red_U/m1 [Consulta: 2016, 15 de mayo].

Muñoz, C. (2003). Segundas lenguas. Adquisición en el aula. IBÉRICA, 6, 155-169.

Torrelles Nadal, C., Coiduras Rodríguez, J. L., Isus, S., Carrera, X., París Mañas, G., \& Cela, J. M. (2011). Competencia de trabajo en equipo: definición y categorización. Profesorado: revista de currículum y formación del profesorado, 2011, (15-3), 329-344.

Valero, M., Aramburu, J., Baños, J. E., Sentí, M., \& Pérez, J. (2007). Introducción de un portafolio para fomentar competencias transversales de los estudiantes universitarios. Educación Médica, 10(4), 50-57.

Vieira, M. J., \& Vidal, J. (2006). Tendencias de la Educación Superior europea e implicaciones para la orientacion umversitaria $=$ Trends in European Higher Education and implications for student support and guidance. REOP-Revista Española de Orientación y Psicopedagogía, 17(1), 75-97.

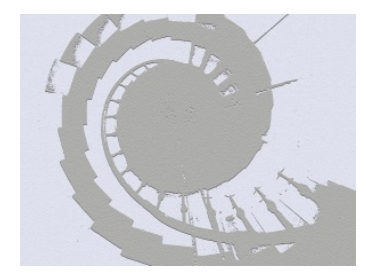

